AMERICAN JOURNAL OF SOCIAL AND MANAGEMENT SCIENCES

ISSN Print: 2156-1540, ISSN Online: 2151-1559, doi:10.5251/ajsms.2011.2.3.316.324

(C) 2011, ScienceHuß, http://www.scihub.org/AJSMS

\title{
Under pricing in initial public offering
}

\section{Eddy Junarsin}

Faculty of Economics and Business, Universitas Gadjah Mada

\begin{abstract}
This study examines the underpricing in initial public offering (IPO). In some years, the means of gross proceed and money left on the table of after-merging data exceed those of beforemerging data. The vast majority of IPO firms employed highly reputable underwriters (reputation between 6 and 9) during the period of 2000-2009. Average gross proceed is also much higher in IPO cases where the lead underwriter is reputable. Likewise, initial return and money left on the table are mostly and substantially higher for the case of reputable lead underwriter, thereby supporting the conjecture that IPO firms do prefer a reputable lead underwriter to get better analyst coverage although they have to sacrifice money left on the table. Financial firms that undergo IPO are also on average older. Results suggest that log of age and 36-month cumulative market geometric return positively and significantly affect the long-term performance of IPO securities.
\end{abstract}

Keywords: JEL classifications: G12, G24, initial public offering, underpricing, underwriter.

\section{INTRODUCTION}

This paper examines the underpricing in initial public offering (IPO) that has been documented in vast array of literature. In addition, several variables expected to influence the underpricing are analyzed, such as industry, age, market return, initial return (first trading day return), and IPO volume in the issuing year. Databases utilized comprise IPOSCOOP, Ritter's data, and CRSP.

Brief Literature: Loughran and Ritter (2004) tested three hypotheses: (1) the changing risk composition, (2) the realignment of incentives, and (3) the changing issuer objective function. Changing risk composition hypothesis argues that IPO underpricing, which was very obvious in early 2000 s, is influenced by the fact that high risk companies increasingly go into the market through IPOs. Realignment of incentives hypothesis suggest that IPO is underpriced since executives have low ownership in the company before going public. Meanwhile, changing issuer objective function hypothesis says that firm managers are willing to lose money left on the table in order to get more analyst coverage. This leads them to pay high prices directly and indirectly to reputable underwriters. Loughran and Ritter (2004) find that the changing issuer objective function hypothesis can explain the IPO underpricing phenomenon better than can other hypotheses.
IPOSCOOP Data: This database provide data on trade date, company name, ticker, underwriters, reputation, number of shares, gross proceeds, offer price, opening price, first-day close price, and initial return. Data available are from May 2000 to 2009. From these data, I calculate money left on the table as:

Money left on the table $=$ (First-day close price Offer price) / Number of shares

1

Ritter's Data: From Ritter's website (http://bear.warrington.ufl.edu/RITTER/ipodata.htm), I get additional data, such as reputation and founding year of each IPO company. Having the founding year data, I can estimate the age of a firm on its IPO day by subtracting founding year from IPO year. This database also has offer date and company name, which would help in the merging process with IPOSCOOP data. In order to match IPOSCOOP data, I only use Ritter's data from 2000 to 2009. Loughran and Ritter (2004) argue that an underwriter with reputation of 8 or 9 is considered a nationally reputable underwriter whereas that with reputation of 6 or 7 is a regionally recognized underwriter. Hence, I follow their approach by creating a dummy variable taking value of 1 if the underwriter has reputation between 6 and 9 , and 0 otherwise. I handmatch Ritter's reputation data one by one with IPOSCOOP data. Another advantage offered by Ritter's database is the availability of PERMNO, which would benefit me in merging IPOSCOOP-Ritter data with CRSP data later. 
CRSP Data: From CRSP database, data collected include daily PERMNO, trading date, security return, SIC code, and market return from 2000 to 2009. As also done in previous exercise, I categorize SIC based on two-digit SIC code as follows:

Data Analysis: Tables 2 and 3 depict the descriptive statistics of data before and after merging process. After merging, total observations decrease to 784 IPOs from 1,549 IPOs before merging. Year-by-year comparison indicates that the after-merging data cover between $40 \%$ and $63 \%$ of the before-merging data. In some years, the means of gross proceed and money left on the table of after-merging data exceed those of before-merging data.

Table 4 describes mean and median of final sample. Median figures are much more moderate than mean data, indicating that data are skewed. Meanwhile, almost all of mean data are significantly different from zero.

Table 5 shows the mean by year and reputation. It can be noticed that the vast majority of IPO firms employed highly reputable underwriters (reputation between 6 and 9) during the period of 2000-2009. Average gross proceed is also much higher in IPO cases where the lead underwriter is reputable. Likewise, initial return and money left on the table are mostly and substantially higher for the case of reputable lead underwriter, thereby supporting the conjecture that IPO firms do prefer a reputable lead underwriter to get better analyst coverage although they have to sacrifice money left on the table. Age group of $>15$ years experiences the highest gross proceed and money left on the table while age group of 5-10 years has the highest day-0 return

Table 1. Categorization of SIC Code (U.S. Department of Labor 2010)

\begin{tabular}{|l|l|}
\hline \multicolumn{1}{|c|}{ Two-Digit SIC Code } & \\
\hline 01 to 09 & Agriculture, Forestry, and Fishing \\
\hline 10 to 14 & Mining \\
\hline 15 to 19 & Construction \\
\hline 20 to 39 & Manufacturing \\
\hline 40 to 49 & Transportation, Communications, Electric, Gas, and Sanitary Services \\
\hline 50 to 51 & Wholesale Trade \\
\hline 52 to 59 & Retail Trade \\
\hline 60 to 69 & Finance, Insurance, and Real Estate \\
\hline 70 to 90 & Services \\
\hline 91 to 99 & Public Administration \\
\hline
\end{tabular}

Table 2. Means of Data before and after Merging

\begin{tabular}{|r|r|r|r|r|r|r|r|r|}
\hline Year & \multicolumn{4}{|c|}{ IPOSCOOP (Mean) } & \multicolumn{4}{c|}{ After Merging with CRSP (Mean) } \\
\hline & \multicolumn{1}{|c|}{ Obs. } & \multicolumn{1}{|l|}{ Proceed } & \multicolumn{1}{|c|}{ Initial Return } & \multicolumn{1}{l|}{ Left on Table } & \multicolumn{1}{l|}{ Obs. } & \multicolumn{1}{l|}{ Proceed } & \multicolumn{1}{l|}{ Initial Return } & \multicolumn{1}{l|}{ Left on Table } \\
\hline 2000 & 237 & 352.4825437 & 0.355621126 & 51161.43091 & 96 & 432.0850375 & 0.269915948 & 30178.84049 \\
\hline 2001 & 94 & 448.7634521 & 0.127612834 & 33197.66523 & 38 & 266.7665132 & 0.141877902 & 31641.54123 \\
\hline 2002 & 82 & 305.5528696 & 0.072315332 & 13245.2906 & 35 & 149.3374274 & 0.071521526 & 13224.58169 \\
\hline 2003 & 80 & 202.4987569 & 0.11944606 & 26811.10863 & 39 & 131.0779714 & 0.137463414 & 15986.32765 \\
\hline 2004 & 232 & 184.8086119 & 0.107032262 & 16496.02727 & 124 & 190.7131392 & 0.126811564 & 22173.13377 \\
\hline 2005 & 225 & 163.6789084 & 0.099387966 & 15274.05959 & 121 & 172.7163647 & 0.114889881 & 18267.14233 \\
\hline 2006 & 237 & 192.8146957 & 0.099884709 & 21487.66427 & 125 & 221.4984316 & 0.110671671 & 26208.58559 \\
\hline 2007 & 256 & 218.7527559 & 0.115484168 & 24475.32682 & 162 & 220.9092388 & 0.126651137 & 25501.26545 \\
\hline 2008 & 46 & 607.5907233 & 0.023046307 & 121895.5726 & 18 & 273.3701817 & 0.02446869 & 26196.1271 \\
\hline 2009 & 60 & 530.2424689 & 0.072947521 & 19091.82989 & 26 & 252.5013485 & 0.068773816 & 17227.59956 \\
\hline
\end{tabular}


Table 3. Comparison between After-Merging Data and Before-Merging Data

\begin{tabular}{|c|c|c|c|}
\hline \multirow[t]{2}{*}{ Year } & \multicolumn{3}{|c|}{ After-Merging Data / Before-Merging Data } \\
\hline & Obs. & Proceed & Left on Table \\
\hline 2000 & $40.51 \%$ & $122.58 \%$ & $58.99 \%$ \\
\hline 2001 & $40.43 \%$ & $59.44 \%$ & $95.31 \%$ \\
\hline 2002 & $42.68 \%$ & $48.87 \%$ & $99.84 \%$ \\
\hline 2003 & $48.75 \%$ & $64.73 \%$ & $59.63 \%$ \\
\hline 2004 & $53.45 \%$ & $103.19 \%$ & $134.41 \%$ \\
\hline 2005 & $53.78 \%$ & $105.52 \%$ & $119.60 \%$ \\
\hline 2006 & $52.74 \%$ & $114.88 \%$ & $121.97 \%$ \\
\hline 2007 & $63.28 \%$ & $100.99 \%$ & $104.19 \%$ \\
\hline 2008 & $39.13 \%$ & $44.99 \%$ & $21.49 \%$ \\
\hline 2009 & $43.33 \%$ & $47.62 \%$ & $90.24 \%$ \\
\hline
\end{tabular}

Table 4. Mean and Median of After-Merging Data

\begin{tabular}{|c|c|c|c|c|c|c|c|c|}
\hline \multirow[b]{2}{*}{ Year } & \multicolumn{4}{|c|}{ Mean } & \multicolumn{4}{|c|}{ Median } \\
\hline & Proceed & Initial Return & Left on Table & Age & Proceed & Initial Return & Left on Table & Age \\
\hline 2000 & 352.4825437 & 0.355621126 & 51161.43091 & 9.3125 & 60 & 0.119791667 & 7687.5 & 7 \\
\hline 2001 & 448.7634521 & 0.127612834 & 33197.66523 & 20.97368421 & 83.25 & 0.094375 & 5660 & 10 \\
\hline 2002 & 305.5528696 & 0.072315332 & 13245.2906 & 19 & 82.5 & 0.027142857 & 760 & 10 \\
\hline 2003 & 202.4987569 & 0.11944606 & 26811.10863 & 12.71794872 & 85 & 0.102941176 & 11550 & 9 \\
\hline 2004 & 184.8086119 & 0.107032262 & 16496.02727 & 18.70967742 & 93.75 & 0.075714286 & 5925 & 9 \\
\hline 2005 & 163.6789084 & 0.099387966 & 15274.05959 & 29.03305785 & 120.19116 & 0.060769231 & 6545 & 10 \\
\hline 2006 & 192.8146957 & 0.099884709 & 21487.66427 & 23.128 & 127.5 & 0.044444444 & 4005 & 10 \\
\hline 2007 & 218.7527559 & 0.115484168 & 24475.32682 & 13.24691358 & 132.4 & 0.059411765 & 7860 & 8 \\
\hline 2008 & 607.5907233 & 0.023046307 & 121895.5726 & 20.22222222 & 188.75 & -0.020555556 & -1175 & 8.5 \\
\hline 2009 & 530.2424689 & 0.072947521 & 19091.82989 & 20.15384615 & 140.360715 & 0.037175926 & 6530.09245 & 10.5 \\
\hline
\end{tabular}

Color in blue means significantly different from zero

Table 5. Mean of Final Sample by Year and Reputation

\begin{tabular}{|c|c|c|c|c|c|c|}
\hline Year & Reputation & Obs. & Proceed & Initial Return & Left of the Table & Age \\
\hline \multirow[t]{2}{*}{2000} & 0 & 2 & 42.8 & 0.1359375 & 5403.125 & 5.5 \\
\hline & 1 & 94 & 440.3676979 & 0.272766553 & 30705.98338 & 9.393617021 \\
\hline \multirow[t]{2}{*}{2001} & 0 & 3 & 69.33333333 & 0.043472222 & 2350 & 6.666666667 \\
\hline & 1 & 35 & 283.6893571 & 0.150312674 & 34152.24477 & 22.2 \\
\hline \multirow[t]{2}{*}{2002} & 0 & 4 & 39.425 & 0.057316964 & 4264 & 22 \\
\hline & 1 & 31 & 163.5196761 & 0.073354372 & 14380.78577 & 18.61290323 \\
\hline \multirow[t]{2}{*}{2003} & 0 & 2 & 14.599995 & 0.060416667 & 1160 & 3.5 \\
\hline & 1 & 37 & 137.3740782 & 0.141628103 & 16787.75077 & 13.21621622 \\
\hline \multirow[t]{2}{*}{2004} & 0 & 16 & 61.35625 & 0.059245603 & 5028.40625 & 9.375 \\
\hline & 1 & 108 & 209.8771228 & 0.136821336 & 24713.09341 & 20.09259259 \\
\hline \multirow[t]{2}{*}{2005} & 0 & 11 & 46.32272727 & 0.190541438 & 5865.727273 & 6.818181818 \\
\hline & 1 & 110 & 185.3557285 & 0.107324726 & 19507.28384 & 31.25454545 \\
\hline \multirow[t]{2}{*}{2006} & 0 & 8 & 49.0904525 & 0.122645833 & 8917.382373 & 8.125 \\
\hline & 1 & 117 & 233.2870114 & 0.109852925 & 27390.89008 & 24.15384615 \\
\hline \multirow[t]{2}{*}{2007} & 0 & 11 & 114.8244046 & 0.01390871 & 4513.5357 & 28 \\
\hline & 1 & 151 & 228.6372731 & 0.134864161 & 27030.17291 & 12.17218543 \\
\hline \multirow[t]{2}{*}{2008} & 0 & 1 & 5.775 & -0.047619048 & -275 & 9 \\
\hline & 1 & 17 & 289.1110748 & 0.028709145 & 27753.25222 & 20.88235294 \\
\hline \multirow[t]{2}{*}{2009} & 0 & 1 & 25 & -0.06 & -1500 & 3 \\
\hline & 1 & 25 & 261.6014025 & 0.073924768 & 17976.70354 & 20.84 \\
\hline
\end{tabular}


Table 6. Mean and Median of Final Sample by Age Group

\begin{tabular}{|c|c|c|c|c|c|c|c|c|c|}
\hline \multirow[b]{2}{*}{ Age Group } & \multicolumn{5}{|c|}{ Mean } & \multicolumn{4}{|c|}{ Median } \\
\hline & Obs. & Proceed & Initial Return & Left of the Table & Age & Proceed & Initial Return & Left of the Table & Age \\
\hline $0-5$ & 176 & 159.9346576 & 0.094716653 & 13660.87814 & 2.176136364 & 114.3 & 0.016491228 & 1712.5 & 2 \\
\hline $5-10$ & 259 & 255.6604343 & 0.173404202 & 24920.89104 & 6.980694981 & 80.1 & 0.0966666667 & 6400 & 7 \\
\hline $10-15$ & 114 & 137.5477767 & 0.149413258 & 23345.26185 & 11.71929825 & 85 & 0.088972498 & 7800 & 12 \\
\hline$>15$ & 235 & 303.90873 & 0.113868065 & 29583.86424 & 47.16170213 & 161.1111095 & 0.058823529 & 9375 & 33 \\
\hline
\end{tabular}

Color in blue means significantly different from zero

Table 7. Mean and Median of Final Sample by SIC Category

\begin{tabular}{|c|c|c|c|c|c|c|c|c|c|}
\hline \multirow[b]{2}{*}{ SIC Cat. } & \multicolumn{5}{|c|}{ Mean } & \multicolumn{4}{|c|}{ Median } \\
\hline & Obs. & Proceed & Initial Return & Left of the Table & Age & Proceed & Initial Return & Left of the Table & Age \\
\hline 0 & 579 & 211.4113056 & 0.119193045 & 23966.1629 & 20.69602763 & 121.5 & 0.060769231 & 7370 & 9 \\
\hline 1 & 1 & 140 & -0.066 & -9240 & 15 & 140 & -0.066 & -9240 & 15 \\
\hline 2 & 1 & 80 & 0.039375 & 3150 & 23 & 80 & 0.039375 & 3150 & 23 \\
\hline 3 & 1 & 40 & 0 & 0 & 28 & 40 & 0 & 0 & 28 \\
\hline 4 & 81 & 81.22128884 & 0.24823897 & 26735.63426 & 11.86419753 & 52 & 0.102678571 & 4500 & 8 \\
\hline 5 & 18 & 267.8611116 & 0.134539475 & 27716.69811 & 11.94444444 & 120 & 0.051785714 & 4300 & 5.5 \\
\hline 6 & 3 & 162.0916667 & 0.032457265 & 7605.083333 & 11 & 121.875 & 0.011538462 & 1406.25 & 8 \\
\hline 7 & 7 & 73.55928571 & 0.106507693 & 20987.13456 & 18.71428571 & 42.89 & 0.00375 & 90 & 15 \\
\hline 8 & 12 & 398.848543 & 0.081767982 & 52423.11412 & 30.66666667 & 104.98 & 0.075259875 & 9521.8082 & 10 \\
\hline 9 & 80 & 519.4945306 & 0.150983586 & 14261.86507 & 10.6 & 61.4786355 & 0.078685897 & 5040 & 7.5 \\
\hline 10 & 1 & 49.045 & -0.044117647 & -2163.75 & 5 & 49.045 & -0.044117647 & -2163.75 & 5 \\
\hline
\end{tabular}

Services industry gains the highest gross proceed and initial return whereas financial industry suffers the most from money left on the table. Financial firms that undergo IPO are also on average older.

Table 8. Mean and Median of Final Sample by Gross Proceed Rank

\begin{tabular}{|c|c|c|c|c|c|c|c|c|c|}
\hline \multirow[b]{2}{*}{ Proceed Rank } & \multicolumn{5}{|c|}{ Mean } & \multicolumn{4}{|c|}{ Median } \\
\hline & Obs. & Proceed & Initial Return & Left of the Table & Age & Proceed & Initial Return & Left of the Table & Age \\
\hline 1 & 195 & 37.95141484 & 0.085173691 & 3435.174275 & 11.56410256 & 40 & 0.020833333 & 760 & 8 \\
\hline 2 & 197 & 80.11353865 & 0.166875069 & 13630.51189 & 12.89847716 & 79.992 & 0.097647059 & 8300 & 8 \\
\hline 3 & 196 & 144.2082098 & 0.159818804 & 22561.78082 & 21.46428571 & 139.035715 & 0.094047619 & 11899.6875 & 10 \\
\hline 4 & 196 & 663.3461641 & 0.125337664 & 54567.42048 & 28.60714286 & 374.5 & 0.0525 & 18531.25 & 12.5 \\
\hline
\end{tabular}

I create four ranks based on gross proceed. Table 8 shows that second rank group gets the highest initial return and has the most money left on the table.
Meanwhile, rank-4 group (the highest gross proceed group) is comprised of older firms on average 
Table 9. Mean and Median of Final Sample Using Several Types of Returns

\begin{tabular}{|r|r|r|r|r|r|r|r|}
\hline & & \multicolumn{3}{|c|}{ Mean } & \multicolumn{2}{c|}{ Median } \\
\cline { 3 - 8 } Year & \multicolumn{1}{|c|}{ Obs. } & \multicolumn{1}{|c|}{ Initial Ret. } & \multicolumn{1}{|c|}{ Market-adj. Ret. } & \multicolumn{1}{c|}{ Ind-adj. Ret. } & \multicolumn{1}{c|}{ Initial Ret. } & \multicolumn{1}{c|}{ Market-adj. Ret. } & \multicolumn{1}{c|}{ Ind-adj. Ret. } \\
\hline 2000 & 96 & 0.269915948 & 0.272091585 & $8.3845 \mathrm{E}-18$ & 0.119791667 & 0.125143036 & -0.093536754 \\
\hline 2001 & 38 & 0.141877902 & 0.140379662 & $8.76492 \mathrm{E}-18$ & 0.094375 & 0.087725649 & \\
\hline 2002 & 35 & 0.071521526 & 0.073097994 & $-5.55112 \mathrm{E}-18$ & 0.027142857 & 0.030868268 & 0.017744963 \\
\hline 2003 & 39 & 0.137463414 & 0.137272454 & $8.54018 \mathrm{E}-18$ & 0.102941176 & 0.114221796 & -0.021040981 \\
\hline 2004 & 124 & 0.126811564 & 0.125939621 & $-1.54446 \mathrm{E}-17$ & 0.075714286 & 0.069505148 & -0.042292593 \\
\hline 2005 & 121 & 0.114889881 & 0.113594915 & $-4.03717 \mathrm{E}-17$ & 0.060769231 & 0.056612049 & -0.048022618 \\
\hline 2006 & 125 & 0.110671671 & 0.11048756 & $4.64073 \mathrm{E}-17$ & 0.044444444 & 0.046179007 & -0.064213745 \\
\hline 2007 & 162 & 0.126651137 & 0.127847427 & $6.12936 \mathrm{E}-17$ & 0.059411765 & 0.05906188 & -0.064901016 \\
\hline 2008 & 18 & 0.02446869 & 0.027961757 & $1.86965 \mathrm{E}-17$ & -0.020555556 & -0.010424124 & -0.031448873 \\
\hline 2009 & 26 & 0.068773816 & 0.066667561 & $2.66881 \mathrm{E}-18$ & 0.037175926 & 0.025719234 & -0.03159789 \\
\hline
\end{tabular}

In Table 9, several measures of returns are harnessed.

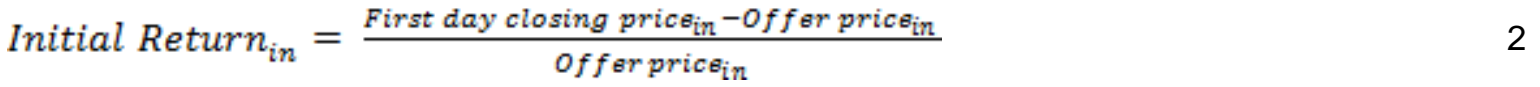

Market adjusted return $_{i n}=$ First day closing price $_{\text {in }}-$ Market return $_{n} \quad 3$

Industry adjusted return $_{i n}=$ First day closing price $_{i n}-$ Average industry return ${ }_{s n}$

for each firm i, IPO event $\mathrm{n}$, and SIC category s.

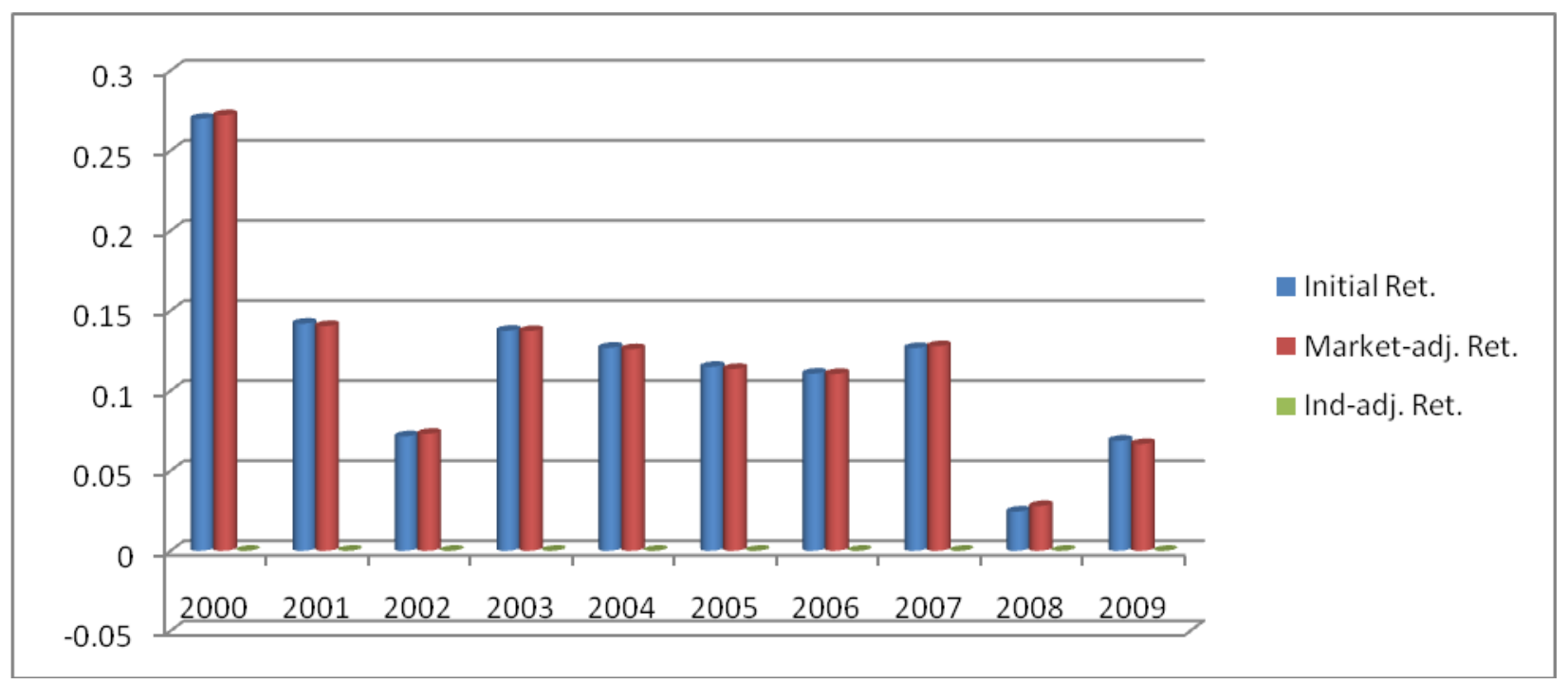

Figure 1. Comparison among Returns

It is shown that initial return and market return are almost identical whereas industry-adjusted return is much lower.

The following tables and figures show various returns, such as arithmetic, geometric, marketadjusted, and industry-adjusted returns. For arithmetic return, the following formulae are utilized:
Cumulative return $_{i t}=\sum_{t=1}^{36}$ Return $_{i t}$ 5 Average Cumulative return $n_{t}=\frac{\sum_{i=1}^{l} \text { Cumulative Return }_{\text {it }}}{I}$ for firm $i=1$ to $I$, month $t=1$ to 36 . 
Table 10. Arithmetic Return

\begin{tabular}{|c|c|c|c|c|}
\hline Month & Obs. & Return & p-value & Cum. Ret. \\
\hline 1 & 16391 & 0.000864282 & 0.009941268 & 0.000864282 \\
\hline 2 & 16215 & -0.000378976 & 0.251990402 & 0.000485306 \\
\hline 3 & 16073 & 0.000358653 & 0.288272804 & 0.000843959 \\
\hline 4 & 16037 & -0.000522179 & 0.127482327 & 0.00032178 \\
\hline 5 & 16017 & $-3.01723 \mathrm{E}-05$ & 0.937455656 & 0.000291608 \\
\hline 6 & 15987 & -0.000563341 & 0.144925911 & -0.000271734 \\
\hline 7 & 15952 & -0.000163343 & 0.624654546 & -0.000435077 \\
\hline 8 & 15893 & $5.9598 \mathrm{E}-05$ & 0.875972099 & -0.000375479 \\
\hline 9 & 15855 & 0.000769522 & 0.029183515 & 0.000394043 \\
\hline 10 & 15792 & -0.00030638 & 0.393910258 & 8.76635E-05 \\
\hline 11 & 15743 & $-6.87647 \mathrm{E}-05$ & 0.8441736 & $1.88988 \mathrm{E}-05$ \\
\hline 12 & 15626 & -0.000477912 & 0.241119825 & -0.000459013 \\
\hline 13 & 15498 & 0.000505549 & 0.217814277 & 4.65358E-05 \\
\hline 14 & 15409 & $-2.29655 \mathrm{E}-05$ & 0.952856534 & 2.35703E-05 \\
\hline 15 & 15350 & 0.000325694 & 0.42578395 & 0.000349264 \\
\hline 16 & 15228 & 0.000778992 & 0.060481917 & 0.001128256 \\
\hline 17 & 15103 & 0.00091568 & 0.024782229 & 0.002043936 \\
\hline 18 & 14940 & 0.000286723 & 0.47027772 & 0.002330659 \\
\hline 19 & 14858 & 0.00051958 & 0.178088021 & 0.002850239 \\
\hline 20 & 14743 & 0.00018659 & 0.647409683 & 0.003036829 \\
\hline 21 & 14570 & -0.000138644 & 0.728533244 & 0.002898184 \\
\hline 22 & 14442 & 0.000453597 & 0.286257809 & 0.003351781 \\
\hline 23 & 14299 & 0.000390398 & 0.33775657 & 0.003742179 \\
\hline 24 & 14086 & 0.000407856 & 0.36604561 & 0.004150035 \\
\hline 25 & 13800 & -7.89952E-05 & 0.848046415 & 0.00407104 \\
\hline 26 & 13333 & 0.000685275 & 0.112738472 & 0.004756315 \\
\hline 27 & 12739 & 0.001020285 & 0.018890525 & 0.0057766 \\
\hline 28 & 12540 & 0.000664222 & 0.168639644 & 0.006440822 \\
\hline 29 & 12383 & 0.00064304 & 0.146322696 & 0.007083862 \\
\hline 30 & 11912 & 0.000610491 & 0.185331414 & 0.007694352 \\
\hline 31 & 11553 & 0.000577524 & 0.157480744 & 0.008271876 \\
\hline 32 & 11282 & 0.000981528 & 0.039784438 & 0.009253404 \\
\hline 33 & 10904 & 0.00119823 & 0.013961352 & 0.010451634 \\
\hline 34 & 10622 & 0.001154136 & 0.017577357 & 0.011605769 \\
\hline 35 & 10313 & 0.000900982 & 0.061032982 & 0.012506752 \\
\hline 36 & 9938 & 0.001019233 & 0.037870516 & 0.013525984 \\
\hline
\end{tabular}

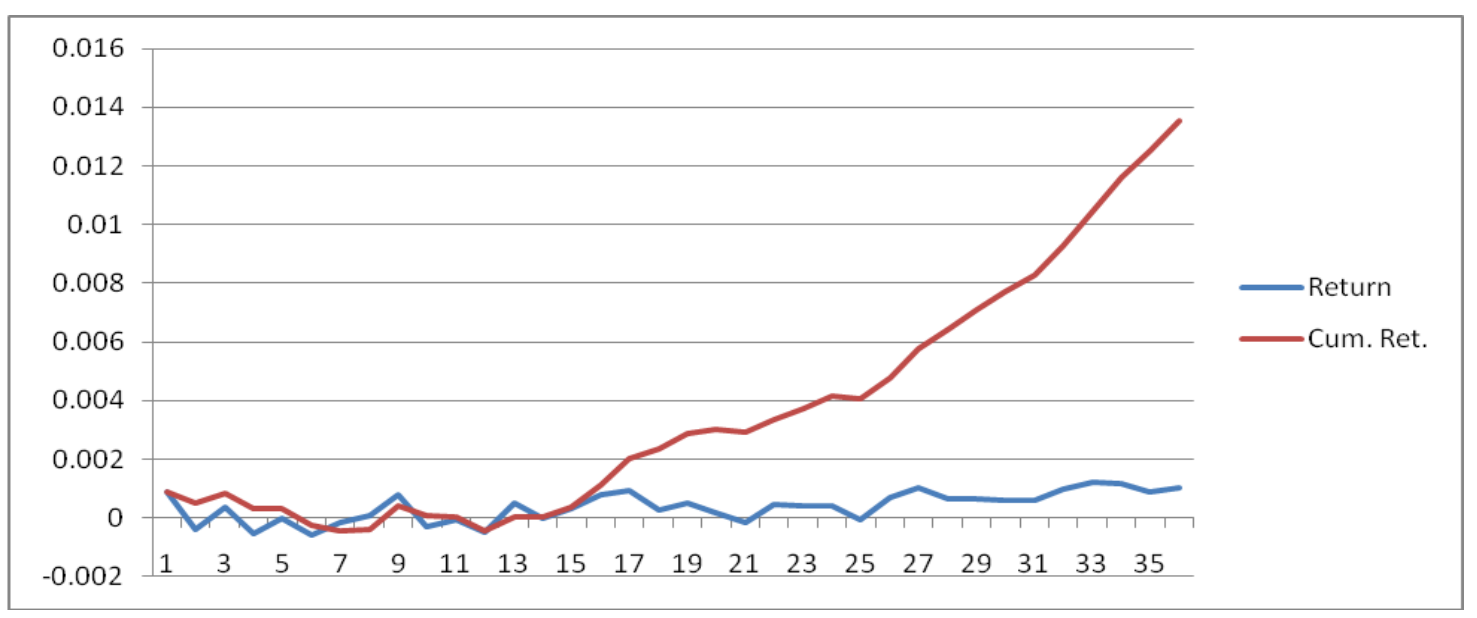

Fig 2. Average Return and Cumulative Return (Arithmetic Return) 
For geometric return, the following formulae are utilized:

Geo return $_{i t}=\left(\prod_{t=1}^{T}\left(1+\operatorname{Ret}_{i t}\right)\right)^{T} \quad 6$

Cumulative geo return $_{i t}=\sum_{t=1}^{36}$ Geometric return $_{i t} \quad 7$

Average cumulative geo return $n_{t}=\frac{\sum_{i=1}^{I} \text { Cumulative geo return }_{i t}}{I} \quad 8$

for firm $\mathrm{i}=1$ to $\mathrm{I}$, month $\mathrm{t}=1$ to 36 .

Table 11. Geometric Return

\begin{tabular}{|c|c|c|c|c|}
\hline Month & Obs. & Geo Ret & p-value & Cum Geo Ret \\
\hline 1 & 780 & $-1.28441 \mathrm{E}-05$ & 0.967571235 & $-1.28441 \mathrm{E}-05$ \\
\hline 2 & 774 & -0.001180728 & 0.000455019 & -0.001193572 \\
\hline 3 & 763 & -0.000487199 & 0.110651897 & -0.001680771 \\
\hline 4 & 762 & -0.001409821 & 6.0317E-05 & -0.003090592 \\
\hline 5 & 760 & -0.001157809 & 0.001979563 & -0.0042484 \\
\hline 6 & 759 & -0.001703991 & 9.50745E-07 & -0.005952391 \\
\hline 7 & 757 & -0.000987355 & 0.009176939 & -0.006939746 \\
\hline 8 & 755 & -0.001021609 & 0.004741873 & -0.007961355 \\
\hline 9 & 753 & -0.00015256 & 0.65981341 & -0.008113915 \\
\hline 10 & 749 & -0.001271082 & 0.000299297 & -0.009384997 \\
\hline 11 & 749 & -0.000960088 & 0.003406853 & -0.010345085 \\
\hline 12 & 744 & -0.001743448 & 2.88626E-05 & -0.012088534 \\
\hline 13 & 740 & -0.001094961 & 0.02795709 & -0.013183495 \\
\hline 14 & 734 & -0.001557167 & 0.004514222 & -0.014740662 \\
\hline 15 & 730 & -0.000886749 & 0.014963223 & -0.015627411 \\
\hline 16 & 726 & -0.000436869 & 0.24639695 & -0.01606428 \\
\hline 17 & 722 & -0.000291075 & 0.425422273 & -0.016355355 \\
\hline 18 & 712 & -0.000796523 & 0.02497323 & -0.017151878 \\
\hline 19 & 708 & -0.000499762 & 0.156303727 & -0.01765164 \\
\hline 20 & 705 & -0.001148506 & 0.011912557 & -0.018800146 \\
\hline 21 & 696 & -0.001250178 & 0.000956316 & -0.020050324 \\
\hline 22 & 690 & -0.000813332 & 0.041864391 & -0.020863655 \\
\hline 23 & 684 & -0.000768061 & 0.06130689 & -0.021631716 \\
\hline 24 & 676 & -0.000850601 & 0.043228212 & -0.022482317 \\
\hline 25 & 663 & -0.001223092 & 0.001936975 & -0.023705409 \\
\hline 26 & 646 & -0.000464805 & 0.278915936 & -0.024170214 \\
\hline 27 & 614 & -0.000346039 & 0.485685012 & -0.024516253 \\
\hline 28 & 599 & -0.000650935 & 0.103110958 & -0.025167189 \\
\hline 29 & 595 & -0.000672102 & 0.164677563 & -0.025839291 \\
\hline 30 & 576 & -0.000621636 & 0.200009931 & -0.026460926 \\
\hline 31 & 559 & -0.000420238 & 0.268968749 & -0.026881165 \\
\hline 32 & 544 & -0.000177283 & 0.676289621 & -0.027058448 \\
\hline 33 & 525 & -0.000109849 & 0.814916509 & -0.027168297 \\
\hline 34 & 511 & 9.15403E-05 & 0.847614561 & -0.027076757 \\
\hline 35 & 497 & -0.000472803 & 0.383533773 & -0.027549561 \\
\hline 36 & 478 & -2.867E-05 & 0.948667769 & -0.027578231 \\
\hline
\end{tabular}




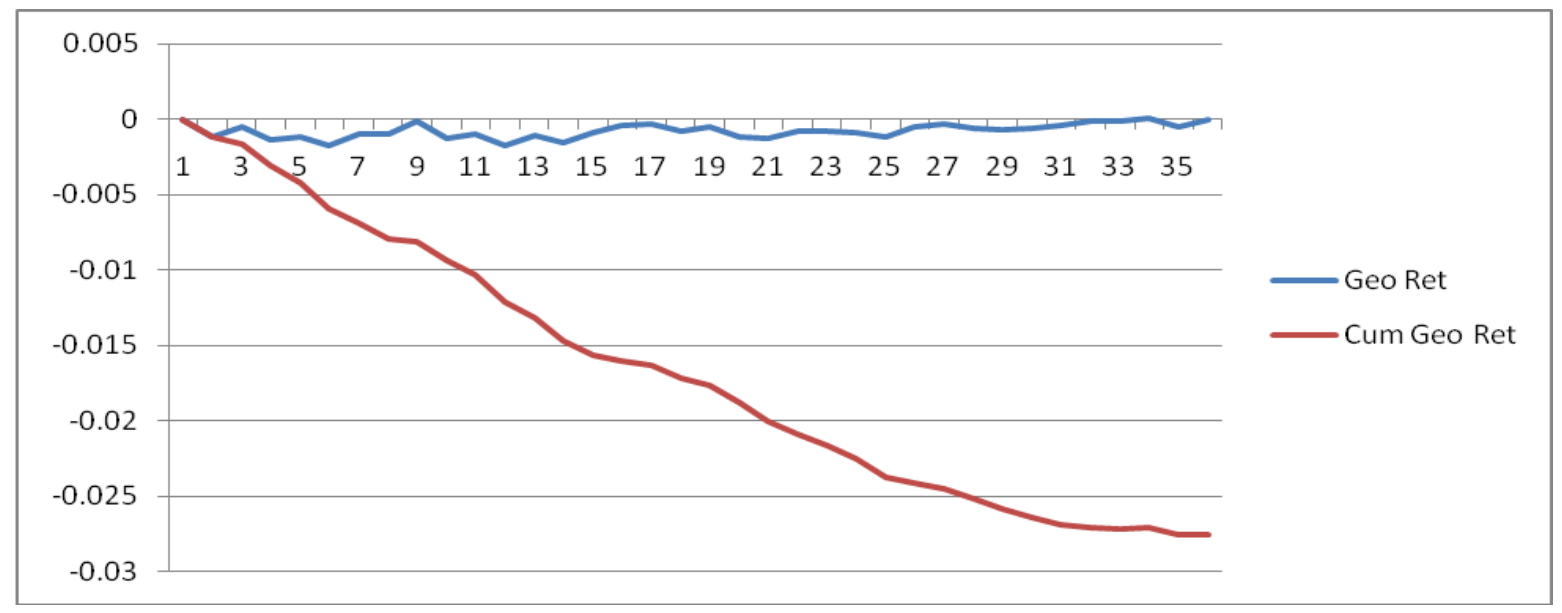

Fig 3. Average Return and Cumulative Return (Geometric Return)

For market-adjusted return, the following formulae are utilized:

Market adj return $_{i t}=\left(\prod_{t=1}^{T}\left(1+\left(\text { Ret }_{i t}-\text { Market ret }_{t}\right)\right)^{T} \quad 9\right.$ Cumulative market adj return $n_{i t}=\sum_{t=1}^{36}$ Market adj return $_{i t} \quad 10$ Average cumulative market adj return $n_{t}=\frac{\sum_{i=1}^{I} \text { Cumulative market adj return } n_{i t}}{I} \quad 11$ for firm $\mathrm{i}=1$ to $\mathrm{I}$, month $\mathrm{t}=1$ to 36 .

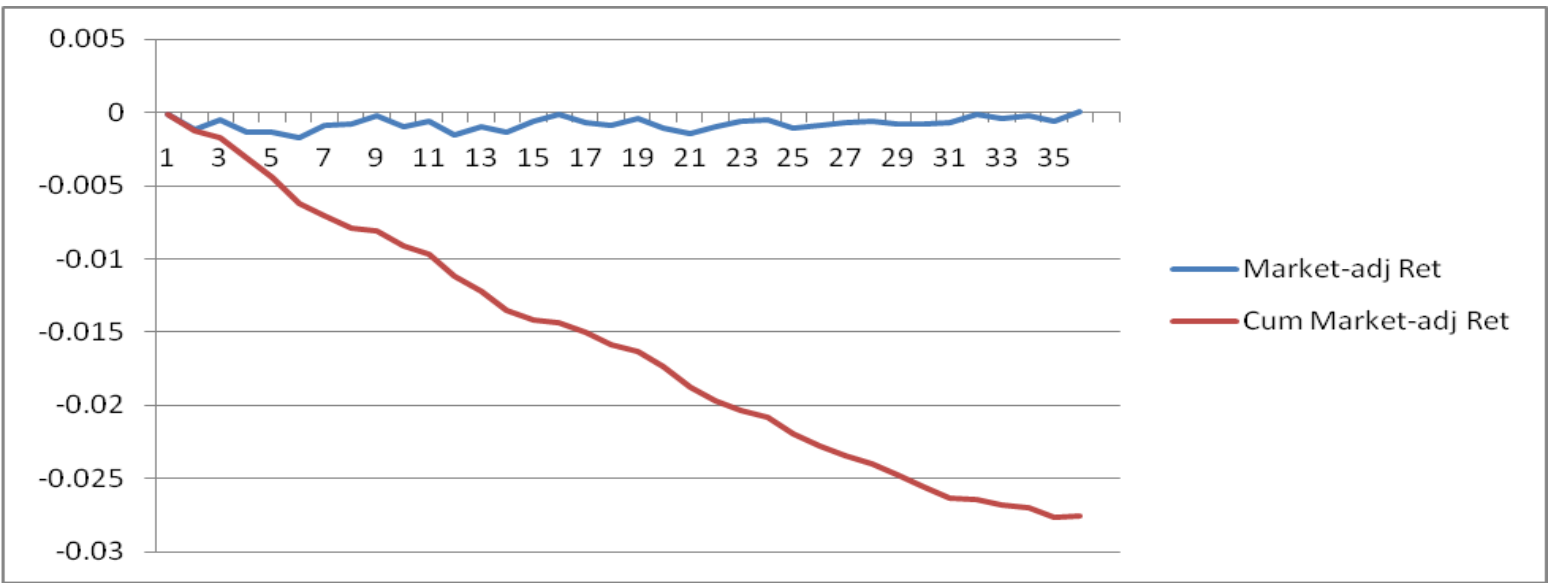

Fig 4. Average Market-adjusted Return and Cumulative Market-adjusted Return

For industry-adjusted return, the following formulae are employed:

Industry adj return it $_{i t}=\left(\Pi_{t=1}^{T}\left(1+\left(\text { Ret }_{i t}-\text { Mean industry ret } t_{s t}\right)\right)^{T} \quad 12\right.$

Cumulative industry adj return $_{i t}=\sum_{t=1}^{36}$ Industry adj return $_{i t} \quad 13$

Average cumulative industry adj return $n_{t}=\frac{\sum_{i=1}^{I} \text { Cumulative industry adj return } n_{i t}}{I} \quad 14$ for firm $\mathrm{i}=1$ to $\mathrm{I}$, month $\mathrm{t}=1$ to 36 , and industry category $\mathrm{s}$. 


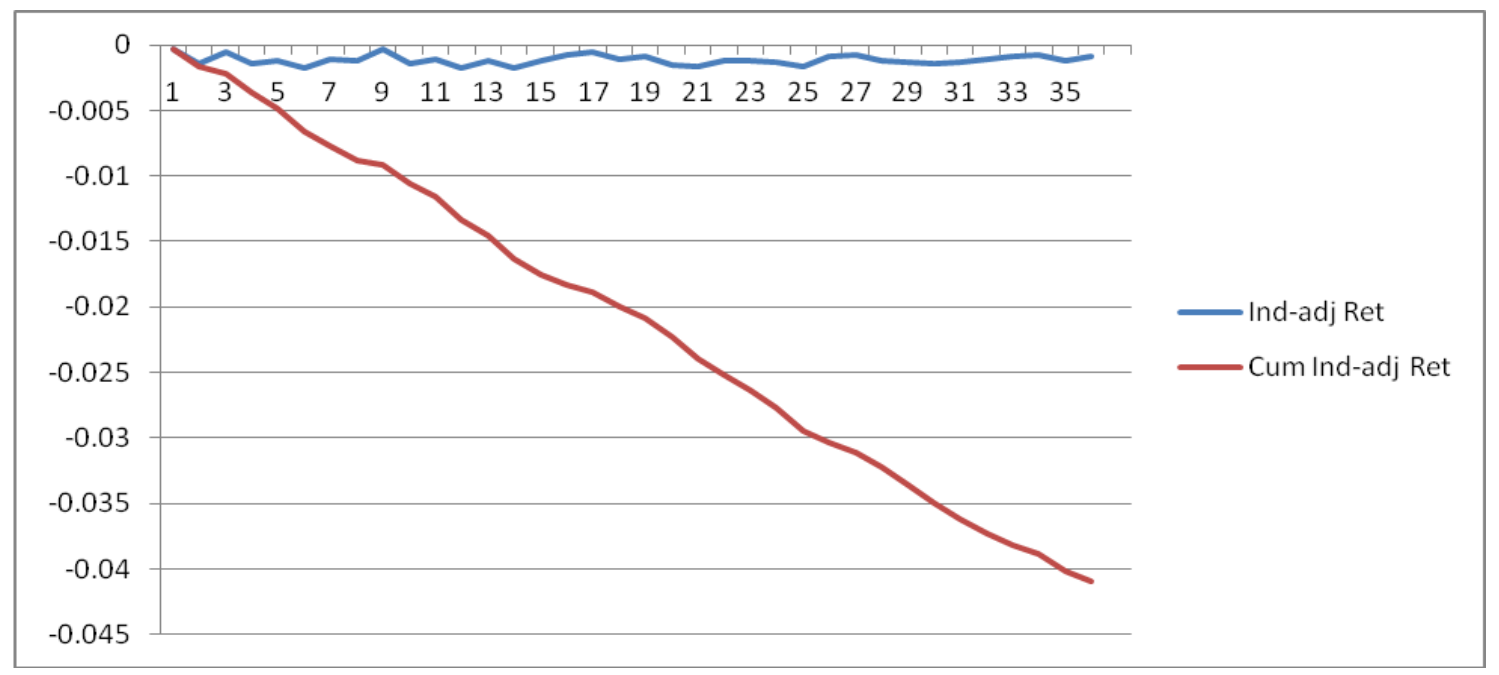

Fig 5. Average Industry-adjusted Return and Cumulative Industry-adjusted Return

Finally, I conduct a regression analysis as those variables or factors analyzed separately above may not necessarily be independent. Regression formula used follows Ritter (1991):

Cumulative geo ret $_{i_{2},=36}=\alpha+\beta_{1}$ Initial Return $_{i, t=0}+\beta_{2} \log (1+\text { Age })_{i, t=0}+$ $\beta_{3}$ Cumulative Market Geo Return $_{i, t=36}$

$\beta_{4}$ No. of IPOs $s_{t=0}+\beta_{5}$ Dummy Mining $i_{i, t=0}+\epsilon$

15

Table 12. Regression Results (Dependent Variable: 36-month Cumulative Geometric Return)

\begin{tabular}{|l|c|c|c|c|c|c|c|}
\hline & Intercept & IR & LogAge & Market Ret & Dmining & Dfinance & No IPOs \\
\hline Parameters & 0.00127923 & 0.00041797 & 0.00012142 & 1.79438331 & 0.00147593 & 0.00044974 & $3.0893 \mathrm{E}-07$ \\
\hline p-value & 0.00016932 & 0.22711476 & 0.10527536 & $2.9643 \mathrm{E}-23$ & 0.51012281 & 0.49448662 & 0.81788295 \\
\hline
\end{tabular}

Results suggest that log of age and 36-month cumulative market geometric return positively and significantly affect the long-term performance of IPO securities. Unfortunately, we cannot examine the reputation effect since the number of observations for non-reputable underwriters is sufficient and unbalanced compared to the reputable underwriters.

\section{CONCLUSION}

In some years, the means of gross proceed and money left on the table of after-merging data exceed those of before-merging data. The vast majority of IPO firms employed highly reputable underwriters (reputation between 6 and 9) during the period of 2000-2009. Average gross proceed is also much higher in IPO cases where the lead underwriter is reputable. Likewise, initial return and money left on the table are mostly and substantially higher for the case of reputable lead underwriter, thereby supporting the conjecture that IPO firms do prefer a reputable lead underwriter to get better analyst coverage although they have to sacrifice money left on the table. Financial firms that undergo IPO are also on average older. Results suggest that log of age and 36-month cumulative market geometric return positively and significantly affect the long-term performance of IPO securities.

\section{REFERENCES}

Loughran, T. and J. Ritter. 1995. The New Issues Puzzle, Journal of Finance 50 (1), p. 23-51.

Loughran, T., J. Ritter, and K. Rydqvist. 1994. Initial Public Offerings: International Insights, Pacific-Basin Finance Journal 2 (2-3), p. 165-199.

Ritter, J. 1991. The Long-Run Performance of Initial Public Offerings, Journal of Finance 46 (1), p. 3-27.

Ritter, J. and I. Welch. 2002. A Review of IPO Activity, Pricing, and Allocations, Journal of Finance 57 (4), p. 1795-1828. 